Cinémas

Revue d'études cinématographiques

Journal of Film Studies

\title{
Le public et la critique. Le difficile récit de la réception
}

\section{Denis Bachand et Lucie Hotte}

Volume 6, numéro 2-3, printemps 1996

La Critique cinématographique

URI : https://id.erudit.org/iderudit/1000973ar

DOI : https://doi.org/10.7202/1000973ar

Aller au sommaire du numéro

Éditeur(s)

Cinémas

ISSN

1181-6945 (imprimé)

1705-6500 (numérique)

Découvrir la revue

Citer cet article

Bachand, D. \& Hotte, L. (1996). Le public et la critique. Le difficile récit de la réception. Cinémas, 6(2-3), 81-99. https://doi.org/10.7202/1000973ar

\section{Résumé de l'article}

Cette étude comparative des discours de réception critique et spectaroriale vise à évaluer à la fois la représentativité de la critique, son incidence sur la réception spectaroriale et les écarts d'interprétation qui différencient ces deux groupes de spectateurs. Les éléments narratifs, thématiques et figuratifs mentionnés dans chacun des discours sont identifiés afin de dégager une image de chacune des instances de réception. Les auteurs fondent leur étude sur l'accueil réservé en France au film Léolo de Jean-Claude Lauzon (1992). 


\title{
Le public et la critique. Le difficile récit de la réception ${ }^{1}$
}

\section{Denis Bachand et Lucie Hotte}

\begin{abstract}
RÉSUMÉ
Cette étude comparative des discours de réception critique et spectatoriale vise à évaluer à la fois la représentativité de la critique, son incidence sur la réception spectatoriale et les écarts d'interprétation qui différencient ces deux groupes de spectateurs. Les éléments narratifs, thématiques et figuratifs mentionnés dans chacun des discours sont identifiés afin de dégager une image de chacune des instances de réception. Les auteurs fondent leur étude sur l'accueil réservé en France au film Léolo de Jean-Claude Lauzon (1992).
\end{abstract}

\section{ABSTRACT}

This comparative study of film critics and spectators' reception aims to evaluate the representativity of critical writing, its influence on the public and the divergences in interpretation between both groups of spectators. The narrative, thematic and figurative elements which constitute both discourses are identified in order to delineate each type of reception. The authors of this article based their study on the reception of JeanClaude Lauzon's Léolo (1992) in France.

D'emblée, les analystes de la réception, autant filmique que littéraire, ont tendance à restreindre la portée de leurs préoccupations. Le champ couvert par l'appropriation des œuvres oblige, en effet, à tenir compte de tellement de variables qu'il est impossible de les considérer toutes simultanément. Voilà pourquoi certains se spécialisent dans l'étude intrinsèque des textes filmiques ou littéraires, alors que d'autres envisagent la question 
du point de vue des discours qui témoignent de la rencontre avec l'œuvre, cette dernière option recouvrant deux territoires: les textes de la critique officielle, que l'on peut retrouver dans les journaux ou les revues spécialisées, et les discours de réception des spectateurs récoltés par le biais d'entretiens semi-directifs.

L'analyse intrinsèque des films ne permet pas de rendre compte à elle seule de l'ensemble de la réception. Elle repère tout au plus certaines structures du texte filmique (que Bordwell nomme cues $^{2}$ ) qui appellent la coopération du spectateur. En fait, elle se fonde toujours sur des hypothèses quant à l'attitude du récepteur, aux compétences mises à l'épreuve par le film et au travail d'interprétation exigé par la structure narrative qui demandent à être vérifiées auprès d'un public concret. Comme le remarque Daniel Dayan: "Le savoir sur un texte, si raffiné soit-il, ne permet pas de prédire l'interprétation qu'il recevra" (p. 150).

L'analyse empirique de la réception par un public non spécialisé comporte elle aussi plusieurs problèmes dont tout premièrement la capacité réelle qu'a le spectateur de rendre compte de sa propre lecture des œuvres. Cette dernière, en effet, est toujours une activité individuelle, intérieure, qui ne se laisse approcher que de façon fragmentaire dans le contexte d'une démarche prudente qui oblige le spectateur à traduire une expérience antérieure qui fait appel à la mémoire. Comme le remarque Gilles Thérien au sujet de la lecture littéraire, "[...] ce que l'on vérifie alors, c'est moins la capacité de lire [ou même la lecture particulière qui est en œuvre] que la capacité de transformer un acte de lecture en acte d'écriture ou en acte de parole» (p. 79). De plus, l'enquête spectatoriale suscite des questions d'ordre méthodologique: dans quelle mesure les enquêtés transforment-ils leur discours pour le rendre conforme à ce qu'ils pensent être les attentes de l'interviewer? Est-ce que l'interviewer oriente indûment le discours de réception par sa façon de poser les questions? Autrement dit, de quelle validité est-il question lorsque l'on aborde la construction a posteriori des interprétations fixées sur un objet de souvenir? Et dans quelle mesure ne doit-on pas plutôt parler de coconstruction de significations dans une dynamique interactionnelle dialogique propice à l'émergence du sens? 
L'analyse des textes critiques éviterait la plupart des écueils que l'on rencontre lors de l'analyse de la réception par des spectateurs. D'abord, les textes critiques sont facilement accessibles et ils n'ont pas à être sollicités. Ensuite, comme le remarque Pierre Véronneau, il est plus aisé d'évaluer la réception du spectateur-critique qui, lui, "[...] doit énoncer son opinion avec le moins d'ambiguïté possible" (p. 97). En effet, ce type de discours, contrairement aux propos tenus par les spectateurs ordinaires sur les films, est structuré à partir de conventions institutionnelles qui déterminent, d'une part, les éléments dont le critique doit tenir compte et, d'autre part, la fonction même du texte qui est d'évaluer le film et, ultimement, de le juger. Par ailleurs, comme les textes critiques ne représentent qu'une faible portion de la réception et qu'ils sont, en quelque sorte, prédéterminés par les conventions du genre, il est difficile d'en tirer des généralisations sur la réception en général. De plus, le contexte institutionnel dans lequel s'inscrit le discours critique constitue son auteur en spectateur sérieux: le critique ne voit pas le film par pur plaisir, mais plutôt dans le but de rédiger un texte qui en rendra compte le plus adéquatement possible, c'est-à-dire en tenant compte des paramètres interprétatifs que ce dernier aura intériorisés à propos de son public-lectorat dont il est lui-même l'un des principaux producteurs.

Ainsi chacune de ces trois approches délimite un aspect particulier de la réception. Si le texte n'existe que dans la mesure où il est reçu, il ne faut toutefois pas oublier que cette réception ne se fait pas ex nibilo mais bien à partir d'inscriptions repérables dans le texte qui font appel aux compétences du récepteur à l'intérieur d'un certain contexte de réception déterminé en partie par la critique qui, parce qu'elle appartient à l'institution et a une fonction évaluative et "pédagogique", fixe souvent la norme en déterminant les canons de la qualité. Dès lors, l'enquête empirique devient une composante essentielle qui permet de rejoindre les individus concrets dans leur acte individuel de réception. Chacun de ces paradigmes de recherche se fonde sur des a priori épistémologiques particuliers qui leur donnent consistance et qui déterminent tout à la fois leurs propres horizons de réponses. 
Reste le rapport toujours problématique entre les critiques et les spectateurs ordinaires. Faute de pouvoir aborder la réception sous tous ses angles à la fois, on pourra commencer par comparer la réception critique à la réception spectatoriale. Il est d'ailleurs étonnant que si peu de théoriciens aient confronté l'analyse des textes critiques à l'analyse de la réception par les spectateurs ordinaires alors qu'ils conçoivent le spectateur spécialisé comme l'instance médiatrice entre le film et le public. La plupart, en effet, considèrent les critiques " $[\ldots]$ comme des distillats des goûts, des préférences du public, comme des témoignages indicateurs des représentations, images, mythes et préjugés qui travaillent l'imaginaire collectif d'une société " (Pérusse, p. 92). Si les théoriciens du cinéma ont tendance à considérer le critique comme la «[...] figure de substitution idéalisée des lecteurs" (Bachand, 1995), les spectateurs quant à eux se montrent plutôt méfiants à leur égard ${ }^{3}$. Lors d'une première enquête auprès du public du Festival du cinéma québécois de Blois sur la réception spectatoriale, $45 \%$ des répondants se disaient en général très peu influencés par le jugement des critiques. On constatait par ailleurs une corrélation entre le degré d'importance qu'on leur accorde et le degré de satisfaction à l'endroit des films ${ }^{4}$. Chez les spectateurs qui se disaient "peu influencés", la cote d'appréciation la plus élevée (très) était de $22 \%$. Elle passe à $13 \%$ chez ceux qui se disaient "très influencés". Autrement dit, plus on se dit influencé et plus on semble critique à l'égard des films et moins on semble les apprécier. Ces résultats nous ont amenés à nous interroger sur le rôle de la critique dans le façonnage des goûts du public. La confrontation des textes de réception critique à des comptes rendus de réception faits par des spectateurs ordinaires nous permettra donc d'évaluer à la fois la représentativité de la critique toujours considérée comme acquise, son incidence sur la réception spectatoriale et les écarts d'interprétation entre ces deux registres discursifs.

\section{Un cas type: Léolo en France}

Nous avons choisi pour cette étude le film Léolo du cinéaste Jean-Claude Lauzon (1992). D'une part, ce choix est motivé par des considérations d'ordre pratique, notamment le fait que 


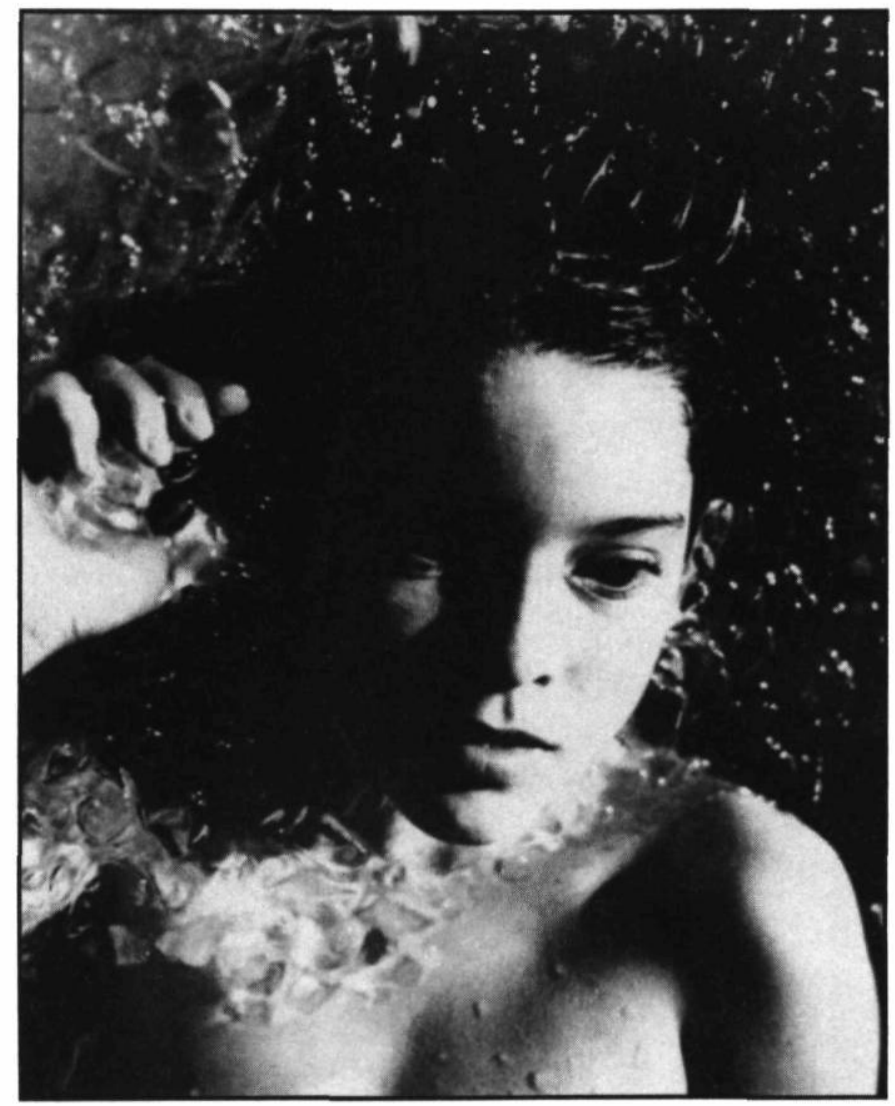

\section{Léolo de Jean-Claude Lauzon (1992)}

Collection Cinémathèque québécoise

notre recherche en cours auprès des spectateurs du Festival du cinéma québécois de Blois nous donnait accès à des "comptes rendus de visionnement " faits par des spectateurs ordinaires. D'autre part, une première analyse de la réception spectatoriale de Léolo laissait entrevoir la rentabilité d'une analyse plus approfondie. Ce film, en effet, à cause de sa structure éclatée et de sa trame narrative complexe, pose aux spectateurs des difficultés particulières lorsqu'ils doivent le raconter et par le fait même souligne de façon exemplaire les éléments qui entrent en jeu lors du processus d'interprétation/réception. Par ailleurs, le fait que la critique journalistique française ait consacré plusieurs articles à Léolo nous a incités à exploiter ce corpus. 
L'intérêt de la critique française pour Léolo est évident. Si quelques articles reprennent le communiqué de presse, la grande majorité des comptes rendus critiques sont originaux. Nous avons donc limité notre corpus critique à 81 articles de journaux parus en France entre le 7 mai et la fin octobre 1992. Notre corpus spectatorial est constitué de 20 entretiens. Les membres du "panel" ont été sélectionnés sur une base de caractérisation sociologique (âge, sexe, profession). Nous avons rencontré les répondants à leur domicile après le visionnement du film au Festival du cinéma québécois de Blois. Nous leur avons alors demandé de raconter le film à une personne de leur entourage qui n'avait pas assisté à la projection (ami-e-s, conjoint-e-s, voisin-e-s).

À partir des textes ainsi compilés, nous avons construit ce que l'on pourrait appeler à la suite de Joly et Soulas (p. 104), qui s'inspirent de Riffaterre, deux "architextes", un pour les critiques et un autre pour les spectateurs. Nous avons retracé dans les divers textes de réception des constantes que nous avons rattachées à trois types d'informations: narratives (qui concernent plus principalement la diégèse), thématiques (commentaires et jugements) et figuratives (jeu des acteurs, images, procédés) sans toutefois retenir d'isotopie comme telle (c'est-à-dire de champ sémantique qui orienterait à l'avance la lecture des textes de réception). Il apparaît par ailleurs que les informations narratives et figuratives servent souvent d'argument pour entériner les commentaires et les jugements. Selon Bordwell (p. 35-37), les arguments seraient de trois types: éthique, esthétique et émotif. Les arguments éthiques attestent l'autorité du critique (ce qui rend son jugement valable), les arguments émotifs font appel aux émotions du spectateur, alors que les arguments esthétiques reflètent les normes qui modèlent les goûts d'un public le plus souvent grâce à des liens que le spectateur établit avec d'autres films qu'il qualifie de bons ou de mauvais. L'hypothèse de départ prend appui sur le fait que le critique, qui a pour but premier d'informer le public lecteur, doit bien connaître ses goûts et ses attentes afin de pouvoir susciter son intérêt. La représentativité du discours critique devrait donc permettre d'établir des filiations entre les deux types de discours. 


\section{Entre le paradis et l'enfer. De l'engouement au dégoût}

La critique est unanime: "Léolo, on ne peut que le détester ou l'avoir dans la peau" (Médioni). Ceux qui ne l'aiment pas, bien que rares parmi les critiques, le détestent ardemment, ceux qui l'aiment, et ils sont nombreux, désirent le revoir. Ce qui est certain, Léolo "[...] ne laisse pas indifférent" (Rémy). Les specțateurs, tout comme les critiques, réagissent de façon fortement émotive à l'œuvre. L'expression "c'est un film qui ne laisse pas indifférent" revient également chez les spectateurs. On signale que "c'est un film qui fait mal». On l'aime et on le déteste parce qu'il choque et qu'il dérange. Léolo choque d'abord parce que l'enfance racontée est "[...] un long cauchemar" (Sotinel) sans aucun rapport avec les stéréotypes qui sont d'ordinaire de mise dans les films sur l'enfance: les "[...] enfants innocents-poètes", "[...] le paradis perdu des passions prépubères" (Lefort) sont absents du film de Lauzon. Les critiques, et à un moindre degré les spectateurs ${ }^{5}$, ne semblent capables de concevoir l'enfance qu'idyllique au sein d'une atmosphère calfeutrée. Or, dans Léolo, l'enfance leur apparaît d'abord et surtout comme une descente en enfer (ce cliché est d'ailleurs parodié par Michel Boujut qui qualifie le film de "descente en enfance" et Sotinel, "plongée dans le noir enfer des souffrances enfantines", de même que dans Femme Actuelle: "Chute en enfer"). La référence à l'enfer apparait aussi dans le discours des spectateurs. On dit que la vie de Léolo "est une descente aux enfers", que c'est "une vie d'enfer".

Pour les critiques, cette descente infernale est constamment contrebalancée par toute la dimension poétique et esthétique qu'ils reconnaissent à l'ensemble du film. C'est grâce à cet entrelacs poésie-cauchemar qu'ils ressentent une invitation au voyage vers leur propre jeunesse: "Sitôt sorti du film, on a envie de revenir aux odeurs, aux peurs et aux manques de notre propre enfance. Envie de faire une grande balade au-dedans de soi, ce lieu si peu fréquenté par le cinéma et pourtant si étrangement visitable" (Jean-Jacques Bernard). Pourtant, cette invitation est contrecarrée par la puissance des clichés qui les habitent depuis si longtemps. Ils se trouvent alors dans une impasse, celle de réconcilier l'enfance stéréotypée avec cette vision crue d'un 
lucide-ingénu que leur offre Jean-Claude Lauzon. Comme le dit si bien Jérôme Mallien : "Léolo, c'est d'abord une voix off, omniprésente, une voix d'adulte qui parle pour un corps d'enfant". Cela choque tellement que Thierry Jousse, qui n'a pas pu opérer la réconciliation, définit Léolo comme "[...] un film qui réussit l'exploit de montrer la vie mentale d'un enfant sans posséder un gramme d'enfance".

C'est l'univers sordide dans lequel vit Léolo qui rend son enfance si cauchemardesque, si dantesque aux yeux des critiques et des spectateurs. Dans leur résumé de l'histoire, les critiques retiennent surtout les scènes dérangeantes, éprouvantes: celle de la masturbation dans le foie de veau, celles de la pendaison du grand-père ou de la noyade de Léolo, celles de la défécation. En fait, le film dit et montre tout ce qui doit être tu. Il met à jour tous les interdits: la merde, la folie, les côtés déplaisants de l'apprentissage de la sexualité (viol du chat, masturbation dans le foie de veau), le désir de tuer. Cette famille étrange, sa pauvreté mais surtout sa folie dérangent certes, mais il semble que la tendresse que les membres de la famille éprouvent les uns envers les autres dérange également. Si elle permet d'une part de rendre le film acceptable aux yeux des critiques, il n'en demeure pas moins qu'elle étonne parce qu'elle relève d'une logique différente. La tendresse et la poésie entrent en contradiction avec les éléments scatologiques et avec le grotesque. La poésie n'est pas de mise dans une réalité aussi sordide. Ce paradoxe expliquerait en partie l'ambivalence des critiques qui hésitent à recommander le film qui leur plaît malgré tout, qui les envoûte et les fascine, sans doute à cause de la facture esthétique qui fait en sorte que le film transcende le sacrilège.

Dans leur résumé du film, les spectateurs quant à eux s'étendent sur les scènes qui les ont le plus surpris ou choqués. Cette façon de raconter le film à partir de séquences ou de scènes est similaire à celle des critiques. Cependant, les scènes retenues par les spectateurs ne sont pas nécessairement les mêmes. Ainsi, presque tous mentionnent les deux scènes où Fernand se fait battre alors qu'il n'y a qu'un seul critique qui s'y attarde. Par contre, les scènes de tentatives de meurtre - celle de la pendaison du grand-père et celle de la noyade de Léolo - apparaissent 
autant dans les résumés des spectateurs que dans ceux des critiques. Le côté infernal de l'univers diégétique avec ses scènes choquantes incite peu les spectateurs à recommander le film. Même ceux qui l'ont beaucoup apprécié, tout comme les critiques d'ailleurs, vont affirmer que ce n'est pas un film pour tous: "Maintenant, c'est un film qui m'a plu personnellement, j'ai envie d'envoyer plutôt des adultes le voir [...] je ne sais pas si je le recommanderais à des enfants et des adolescents, parce que c'est quand même une vision toujours, un univers à la limite de la monstruosité." "Il y a dans Léolo une surenchère du choquant et du grotesque qui relève d'une espèce de logique du "plus" qui vise à hausser le seuil d'acceptabilité.

Les efforts de Léolo pour échapper à la malédiction familiale sont souvent invoqués comme l'élément qui rend le film fascinant. Son combat pour échapper au destin héréditaire touche la critique. On s'attarde à ses armes de prédilection. D'abord, le rêve qui fait contrepoids à la réalité sordide, au cauchemar. Le leitmotiv qui scande le film: "Parce que je rêve, je ne le suis pas" est cité par la grande majorité des critiques et des spectateurs. La plupart retiennent l'invraisemblable ascendance italienne que Léolo se donne et son amour pour Bianca. On apprécie particulièrement le côté éminemment poétique des images qui se rattachent au rêve: les scènes italiennes, la lumière qui émerge du placard. Cependant, du point de vue quantitatif, les critiques comme les spectateurs ne consacrent qu'une infime partie de leur propos à ces scènes: on les mentionne en passant, mais sans vraiment s'y attarder.

Tous les critiques identifient la littérature comme moyen de s'évader de la réalité. Ceux-ci font presque tous état de l'indigence culturelle dans laquelle évolue Léolo. On mentionne le fait que " $[\ldots]$ la lecture et l'écriture $[\ldots]$ font irruption par hasard dans l'appartement surpeuplé» (Sotinel), que «[...] l'unique livre de la maison a une fonction implacable: caler une table" (Médioni). Il est par ailleurs intéressant de voir que l'on s'attarde peu à la lecture comme moyen d'évasion alors que celle-ci est, d'ordinaire, perçue comme une excellente façon de fuir la réalité, de vivre par procuration dans un autre monde. C'est plutôt à l'écriture que les critiques accordent le pouvoir 
d'évasion. Pour certains, l'écriture permet à Léolo de raconter " [...] inlassablement l'histoire de Léolo Lozone, le Rital, son double, son antidote, son garde-fou " (Médioni), donc de se créer une identité autre, de vivre dans un autre monde. Ceci étonne tout de même puisque l'histoire qui nous est racontée en voix off, celle qui choque, nous est présentée comme étant celle écrite par Léolo. Il n'en demeure pas moins que l'écriture permet d'opérer une sorte de transmutation de toute la matière vile (merde, folie, pauvreté) en art/or. Le personnage emblématique du dompteur de vers est cité par la plupart des critiques qui voient en lui l'élément qui permet le passage de la réalité à la fiction. Il agirait en quelque sorte comme la pierre philosophale au creux de l'athanor alchimique, comme le film lui-même résulte d'une transmutation similaire dans la vie de Lauzon. Les spectateurs soulignent, eux aussi, son désir de s'en sortir, de s'évader: "C'est par la poésie, l'écriture et le rêve qu'il s'en sort." En somme, la lecture et l'écriture apparaissent certes comme des moyens de fuir la réalité, mais elles sont aussi une façon de réconcilier poésie et réalité, rêve et cauchemar, condensation et exutoire.

\section{De l'inscription de l'intertextualité à la figure de l'auteur}

Les références à la littérature dans le film ouvrent la porte à un premier réseau intertextuel. La présence du roman de Réjean Ducharme L'Avalée des avalés n'entraîne cependant que peu de références à l'œuvre de cet auteur. Pourtant, lors de sa parution, "L'Avalée des avalés [avait mobilisé], comme aucun autre roman québécois ne l'avait fait auparavant, la critique parisienne" (Gallays, p. 270). Les textes critiques consacrés à Léolo signalent le "[...] parrainage affectueusement revendiqué" (J.-P. L), alors que personne n'établit de véritable parallèle entre les deux œuvres. Certains énoncés de la critique littéraire française relatifs à L'Avalée des avalés laissent cependant entrevoir de nombreuses similitudes. Ainsi Jean Freustié décrit le roman comme un " [...] récit insolite, violent, lyrique, à mi-chemin du rêve et du réel» (p. 46). Si l'on parle peu de Ducharme, il n'en demeure pas moins que l'on fait plusieurs analogies entre le film et divers romans ou écrivains, mais chacun cite un roman ou un auteur différent selon ses préférences personnelles ou son bagage de 
connaissances culturelles, ce qu'Umberto Eco nomme les connaissances encyclopédiques (p. 95-96). Il n'y a pas de consensus à ce sujet. Les références sont variées: chez les critiques, on cite Rabelais pour les éléments scatologiques (Femme Actuelle), Portnoy et son complexe de Philip Roth (Jousse) et Le Mur de Sartre pour la masturbation dans le foie de veau, Bandini de John Fante (G. P.), Les Animals de Bayon (Alain Wais).

Les références cinématographiques sont beaucoup plus homogènes. Le film le plus cité est Toto le héros (Jaco Van Dormael) mais les renvois à La vie est un long fleuve tranquille (Étienne Chatiliez) et à Delicatessen (Jean-Pierre Jeunet et Marc Caro) sont aussi très nombreux. Le rapport se fait surtout sur la base d'une ressemblance anecdotique. Toto le héros et La vie est un long fleuve tranquille, comme Léolo, "[...] parlent de la famille et de l'enfance" (Bernard). Dans chacun de ces films, les héros remettent en question leur appartenance à une famille: dans La vie est un long fleuve tranquille, Maurice et Bernadette ont effectivement été échangés à la naissance alors que dans Toto le héros, Thomas pense avoir subi le même sort et que Léolo s'imagine être l'enfant d'un paysan sicilien via une tomate. Mêmes les critiques qui n'entérinent pas le rapport de ressemblance entre les trois films se sentent obligés de parler de Toto le héros et de La vie est un long fleuve tranquille. Un d'entre eux souligne le fait que même si ces trois films présentent chacun une version d'une même histoire, celle de l'enfance, les deux films européens l'abordent à partir du stéréotype du paradis perdu - "La vie est un long fleuve tranquille (version cynique) [et] Toto le héros (version mou de veau attendri)" (Lefort) alors que dans Léolo, "[...] primo, le paradis est un enfer, et, deuzio, un enfer pavé de mauvaises intentions" (Lefort). Un seul critique pousse la comparaison au-delà du rapport anecdotique. Selon lui, Léolo "[...] provoque le même choc, la même émotion - entre rire et larmes - que Toto le héros. On y trouve le même regard sur l'enfance, la même narration "explosée", récit conduit par une voix off qui juxtapose saynètes tristes ou gaies et se moque de la temporalité" (Ferenczi). Les rapports entre ces films sont malgré tout superficiels, mais ce sont des films connus du public auquel s'adresse la critique, ce qui lui permet de 
donner une idée du film québécois de façon très économe, c'està-dire sans entrer dans une foule de détails. En fait, la référence intertextuelle fonctionne, tout comme l'allusion littéraire d'ailleurs, en misant non pas tant sur les textes ou les films euxmêmes que sur les valeurs symboliques et institutionnelles qui leur ont été assignées par les critiques. Ainsi Toto le héros, La vie est un long fleuve tranquille ont déjà été définis comme des films sur les difficultés de l'enfance, sur l'élaboration d'un roman familial, sur la difficulté de vivre que ressentent certains enfants. Il est avantageux pour la critique d'établir un rapport de ressemblance entre Léolo et ces deux films, car il devient alors inutile de rappeler tous ces détails, supposés déjà connus de leurs lecteurs.

Les spectateurs établissent peu de liens intertextuels sans que l'interviewer intervienne. Malgré tout, Toto le héros et Delicatessen demeurent les films les plus fréquemment cités. Comme les critiques, les spectateurs établissent une analogie entre les deux films sur une base purement diégétique :

Ça m'a rappelé deux films que j'ai adorés, vraiment adorés. Il y a Toto le héros, où alors là, c'est l'usurpation d'identité, c'est-à-dire que ma vie est tellement insupportable qu'il vaut mieux que je sois le fils de quelqu'un d'autre, bon. Ça c'est Toto le héros. Et Delicatessen, c'est le côté boucher du père, qui n'est pas complètement antipathique, mais qui est d'un réaliste un peu plus.

On rapproche aussi assez souvent les tentatives de suicide manquées que l'on retrouve dans Delicatessen aux tentatives de meurtre manquées dans Léolo. L'atmosphère de Léolo fait écho à l'ambiance glauque et morbide de Délicatessen, que l'on compare aux œuvres cinématographiques de Fellini et aux bandes dessinées de Reiser imprégnées de grotesque surréaliste.

$\mathrm{Si}$ les références intertextuelles se font sous forme d'allusions dans le simple but de donner au lecteur une idée du genre de film qu'est Léolo, il en va différemment des renvois à l'auteur et à sa vie. Le caractère autobiographique de l'œuvre est très souvent mentionné par les critiques (et pas du tout par les spectateurs). Ils citent Lauzon qui a décrit son film comme " [...] un grand mensonge à partir de ma vie" (Médioni). Ils signalent 
surtout le fait que Lauzon a vécu dans le même milieu que son héros. Ils s'intéressent à sa venue au monde du cinéma et au rôle qu'a joué André Pêtrowski, qu'on associe au dompteur de vers. L'accent que l'on met sur le caractère autobiographique du film peut être dû aux attentes du public qui veut savoir si le film représente vraiment la vie réelle des gens pauvres de ce quartier de Montréal, mais il souligne surtout le fait que les arguments qu'utilisent les critiques en parlant de Léolo sont de type émotif. En effet, lorsque l'on parle de Jean-Claude Lauzon, son talent de cinéaste est rarement mis de l'avant (ce qui serait un argument esthétique) même si l'on signale qu'il est l'auteur d'Un zoo la nuit; ce qui intéresse la critique c'est, d'abord et avant tout, sa vie, le reste n'est qu'accessoire souvent mentionné entre parenthèses: "Le Québécois Jean-Claude Lauzon (déjà auteur d'Un zoo la nuit qui explorait ses relations difficiles avec son père) plonge en effet sa caméra dans la glaise de son enfance, dans la tourbe de ses souvenirs, dans la boue de ses premiers pas dans la vie." Les spectateurs, pour leur part, ne mentionnent pas le cinéaste probablement parce qu'ils ne le connaissent pas.

\section{Écarts et convergences des instances de réception}

Dans un premier temps, certaines conclusions générales peuvent être tirées de l'analyse comparative des deux types de discours de réception. Ainsi, au niveau narratif, le discours des spectateurs se distingue peu de celui des critiques. Tous deux retiennent et racontent les scènes qui les ont le plus marqués, le plus choqués. Même si les scènes évoquées different, il n’en demeure pas moins qu'elles sont du même genre: dérangeantes, choquantes, fortement émotives. Spectateurs, comme critiques, ne peuvent parler du film en faisant abstraction de leurs sentiments et émotions. Par ailleurs, les énoncés figuratifs sont quasi absents du discours des spectateurs et peu présents dans celui des critiques. Les spectateurs n'ayant pas eu accès aux communiqués de presse et ne connaissant pas à l'avance les acteurs et le cinéaste n'en parlent donc pas alors que les critiques s'attardent surtout sur la vie du cinéaste, séduits par le caractère autobiographique du film que Jean-Claude Lauzon a signalé en entrevue. Quand aux énoncés relatifs au niveau thématique, les 
commentaires et les jugements des critiques rejoignent ceux des spectateurs: on a aimé le film, on a été bouleversé, mais on hésite à le recommander fort probablement de crainte de s'ériger contre la doxa et de paraître "anormal ", "sadique " ou tout simplement de "mauvais goût". Dans le discours des spectateurs, comme dans celui des critiques, les énoncés narratifs et figuratifs sont toujours subordonnés aux énoncés thématiques. Le film semble appeler une prise de position, un jugement de valeur. Le discours de réception puise donc inévitablement dans la rhétorique axiologique.

S'il existe plusieurs similarités entre le contenu des deux discours, le traitement differe. La plus grande partie du discours des spectateurs sert à commenter le film de façon générale en en dégageant le sens profond, à produire des jugements de valeur en particulier en ce qui a trait à la diégèse. Les spectateurs rapportent le contenu de l'œuvre en faisant une large place à leurs propres sentiments, à leurs propres réactions face au film. Vraisemblablement, le spectateur qui raconte le film à un ami ou à un proche tente d'abord d'élucider les sentiments que le film a suscités en lui le plaisir, le dégoût, l'indifférence, l'attrait, avant de reconstituer le récit. Il veut expliquer, donner la source de ces émotions. Son discours est avant tout descriptif et explicatif, presque introspectif. Les arguments qu'il invoque pour entériner son jugement sont donc plutôt de type émotif. On ne parle pas du tout du cinéaste, peu des comédiens et de la technique cinématographique qui pourraient entraîner des arguments de type esthétique. Ainsi, le discours des spectateurs differe de celui des critiques, dans la mesure où les premiers tentent de dépasser le simple compte rendu narratif et se servent de lui pour chercher des sens seconds, pour comprendre le film, alors que les critiques se servent des éléments narratifs surtout pour justifier leur jugement à l'endroit du film. Aussi, le discours critique est-il avant tout descriptif et évaluatif.

Pour le critique, l'important consiste à juger le film et à renforcer ce jugement. Pour ce faire, il doit évidemment faire appel à des éléments descriptifs et c'est pourquoi son discours s'apparente le plus à celui des spectateurs au niveau narratif. Cependant, il ne peut pas se limiter aux seuls arguments émotifs. Le 
discours critique étant considéré comme un discours plus sérieux, étant donné son caractère érudit, doit fournir une base crédible aux jugements. Il est possible que cette contrainte liée au genre discursif soit la raison d'être des énoncés figuratifs. Par ailleurs, le discours critique doit également être concis, alors que le discours des spectateurs n'est pas soumis à cette contrainte. Cette nécessaire concision appelle l'usage de procédés permettant d'évoquer beaucoup en peu de mots. Ainsi, l'usage que les critiques font des renvois intertextuels serait dû à leur fonction allusive: mentionner un titre suffit souvent pour évoquer toute une trame narrative, une atmosphère, un genre.

La plupart des différences entre les deux discours peuvent donc être attribuées au genre auquel ils appartiennent. Le discours critique est un discours sérieux, concis et plus technique alors que celui des spectateurs est un discours entre ami-e-s, nullement régi par des limites de temps, par l'obligation de faire état de certaines choses. Cependant, il demeure une distinction qui ne s'explique pas entièrement par l'appartenance à un genre, c'est le caractère interprétatif qu'a le discours des spectateurs et que n'a pas celui des critiques journalistiques. Plusieurs raisons peuvent expliquer cette différence majeure. Elle peut être davantage due au contexte d'énonciation qu'au type de discours. En présence d'un interviewer auquel il accorde possiblement une compétence supérieure à la sienne dans le domaine du cinéma, le spectateur peut vouloir modeler son discours sur ce qu'il croit être les attentes de l'interviewer. En fait, il ne veut sûrement pas passer pour un spectateur naïf. Il est donc possible qu'il tienne un discours qu'il considère plus "profond" et plus de mise que celui qu'il tiendrait d'ordinaire.

La représentativité de la critique est généralement définie par l'adéquation entre son jugement et celui des spectateurs. Dans le cas de Léolo, la plupart des critiques et des spectateurs s'accordent pour affirmer qu'il s'agit d'un film remarquable qui suscite une vaste gamme d'émotions, qui mérite d'être vu mais pas par n'importe qui. De plus, les raisons qui justifient le jugement des spectateurs sont à peu près les mêmes que celles qu'utilisent les critiques. Il y a donc de nombreuses similarités entre les discours des uns et des autres qui concourent à entériner l'hypothèse 
selon laquelle le discours critique serait représentatif de la réception spectatoriale. Mais cette similitude est-elle due à une incidence réelle du discours critique sur la réception spectatoriale ou ne découlerait-elle pas plutôt de l'appartenance à une même communauté interprétative?

\section{Université d'Ottawa}

\section{NOTES}

1 Cette étude a été réalisée grâce à une subvention du Conseil de recherche en sciences humaines du Canada.

2 David Bordwell dans Narration in the Fiction Film élabore une théorie qui permet d'étudier comment les films sollicitent la coopération des spectateurs dans l'élaboration du film : "The main purpose of this book has been to build a theory of how films, in their formal and stylistic operations, solicit story-constructing and storycomprehending activities from spectators" (London: Methuen, 1985, p. 335).

3 Une étude du magazine Box office de février 1984 accorde aux critiques 3,1\% d'importance dans le choix des films (Voir D. Bachand, "La réception des films québécois en France ", Québec Studies, n 9, 1990, p. 72).

4 Voir Denis Bachand, "Figures de spectateurs. Approche exploratoire du public du Festival du cinéma québécois de Blois", L'Aventure du cinéma québécois en France (collectif). Montréal : XYZ, 1996 (sous presse).

5 Certains d'entre eux affirment que le film est trop près de la réalité.

\section{OUVRAGES CITÉS}

Bachand, Denis. "La réception des films québécois en France». Québec Studies, n” 9 (1990) p. 69-78.

Bachand, Denis. "La réception critique du film Le Déclin de l'empire américain aux États-Unis ». Québec Studies, n 19 (1995) p. 155-168.

Bachand, Denis. "Figures de spectateurs. Approche exploratoire du public du Festival du cinéma québécois de Blois". L'Aventure du cinéma québécois en France (collectif). Montréal. Centre de recherche cinéma/réception de l'Université de Montréal/XYZ, 1996 (sous presse).

Bordwell, David. Making Meaning. Inference in the Interpretation of Cinema. Cambridge: Harvard University Press, 1989.

Bordwell, David. Narration in the Fiction Film. London: Methuen, 1985.

Dayan, Daniel. «Les mystères de la réception ». Le Débat, n 71 (1992) p. 146-162.

Eco, Umberto. Lector in fabula ou la Coopération interprétative dans les textes narratifs. Paris: Grasset, 1985.

Freustié, Jean. "Deux petites filles inexemplaires ». Le Nouvel Observateur (12 novembre 1966).

Gallays, François. "La réception des romans de Ducharme", dans Pierre-Louis Vaillancourt (direction), Paysages de Réjean Ducharme. Montréal: Fides (1994) p. 259-293. 
Joly, Martine et Simone Soulas. "Réception du film et imaginaire du spectateur ". Hors Cadre, n 4 (1986) p. 103-112.

Liebes, Tamar et Elihu Katz. "Six interprétations de la série Dallas». Hermès, n ${ }^{\text {o }} 11$ 12 (1993) p. 125-144.

Pérusse, Denise. «Réception critique et contexte: à propos du Déclin de l'empire américain”. Cinémas, vol. 2, n $\mathrm{n}^{\text {os }}$ 2-3 (1992), p. 89-106.

Thérien, Gilles. "Pour une sémiotique de la lecture». Protée, vol. 18, n 2 (1990) p. 67-80.

Véronneau, Pierre. "Entre le coq et l'aigle: la réception des films de Gilles Carle en France et aux États-Unis». Québec Studies, n 17 (1994) p. 97-105.

\section{CORPUS CRITIQUE}

A., M.-C. « Léolo de Jean-Claude Lauzon ». Le Film français (17 mai 1992).

A., M.-C. "Le lancement de Léolo ». Le Film français (11 septembre 1992).

Achour, Bernard. "L'enfance nue ". Le Cinéphage, n 6 (mai-juin 1992).

B., C. "Rêves de gosse". Le Figaro (18 mai 1992).

B., M. “ Léolo: l'enfant qui rêvait”. Le Républicain lorrain (17 septembre 1992).

Baud, Véronique. "L'enfance toute crue». Paris Normandie (18 septembre 1992).

Bernard, Jean-Jacques. "Voyage au centre de la famille. Léolo". Première (octobre 1992) p. 28.

Bernard, Jean-Jacques. "Jean-Claude Lauzon. L'enfance à vif". Première (octobre 1992) p. 70-71.

Boujut, Michel. "Jean-Claude Lauzon". L'Événement du jeudi (17 au 23 septembre 1992) p. 133.

B[oujut], M[ichel]. «Léolo de Jean-Claude Lauzon avec Maxime Colin, Ginette Reno, Julien Guiomar, Pierre Bourgault ». L'Événement du jeudi (17 au 23 septembre 1992) p. 132.

Bouzet, Ange-Dominique. "Quand un Tarzan sort du "bout" ". Libération (18 mai 1992).

Brialy, Jean-Claude. "Hommage à la mère". Madame Figaro (3 octobre 1992).

C., A. "Léolo, de Jean-Claude Lauzon. Folie et poésie». Les Échos (16 septembre 1992) p. 38.

C., L. "Léolo ". Positif (juillet-août 1992).

Ch., S. “JF, Léolo et Novembre». La Tribune de l'expansion (16 septembre 1992).

Chapron, Pierre. "L'heure des pronostics". Va Matin (18 mai 1992).

"La compétition s'est terminée hier soir. La Croisette attend le palmarès". Le Maine libre (18 mai 1992).

Demangeat, Jean-Loup. "Festival de Cannes. La surprise Léolo». Sud Ouest (18 mai 1992).

Demangeat, Jean-Loup. "L'enfance, avec rage" Sud Ouest (16 septembre 1992).

Deschamps, Hugo. "Envoûtant Léolo». Avantages (octobre 1992).

Dosne, Mathia. "Fantasmes d'un fils de pub" Le Quotidien de Paris (17 septembre 1992).

Duplouy, Jacques. "Cannes 92. Ce soir l'heure de vérité ". Centre-France. La Montagne (18 mai 1992).

"En attendant la Palme». La République du Centre (18 mai 1992). 
Ferenczi, Aurélien. "Les mots de Léolo». 7 à Paris, n 565 (16-22 septembre 1992).

Foret, Béatrice. "Léolo de J.C. Lauzon ". Courrier français (18 septembre 1992).

G., P. "Léolo de Jean-Claude Lauzon ». La Voix du Nord (23 septembre 1992).

Garel, Sylvain. "Léolo de Jean-Claude Lauzon ». Le Courrier de Mantes (16 septembre 1992).

Garel, Sylvain, "Léolo un film de rêve et de folie". Pariscope (7 octobre 1992).

Gasperi, Anne de. "La palme en partage». Le Quotidien de Paris, n 3888 (18 mai 1992).

Heymann, Danièle. “Cannes 92. De l'air, de l'air!». Le Monde (19 mai 1992).

Huleu, Maurice. "Léolo: portrait de famille». Nice-Matin (18 mai 1992).

Jousse, Thierry. "Léolo de Jean-Claude Lauzon". Cahiers du cinéma (juin 1992).

L., J.-P. “Tendre enfance à la dure». L'Humanité (18 mai 1992).

"Les festivaliers attendent le palmarès sur la plage». Centre-France. Le Journal du Centre (18 mai 1992).

Lefort, Gérard. "Léolo, naufragé de l'enfance ". Libération (19 septembre 1992).

Le Morvan, Gilles. "Jean-Claude Lauzon. Léolo». Humanité Dimanche (17 septembre 1992).

"Léolo de Jean-Claude Lauzon ". Le Quotidien de Paris (16 septembre 1992).

"Léolo ". Le Courrier de l'Ouest (23 septembre 1992).

"Léolo ". Femme-Actuelle (7 septembre 1992).

"Léolo ". France-Soir (16 septembre 1992).

"Léolo". Lyon matin (16 septembre 1992).

"Léolo ". Le Nouvel Observateur (17 septembre 1992).

"Léolo de Jean-Claude Lauzon". Le Quotidien du médecin (16 septembre 1992).

"Léolo de Jean-Claude Lauzon". Nord-Matin (16 septembre 1992).

"Léolo en compétition. Souvenirs d'enfance». Le Figaro (16 mai 1992).

"Léolo: foisonnant ". Ouest-France (16 septembre 1992).

"Léolo. Un rire gras et grave". France-Soir (18 mai 1992).

Lequèbe, Éric. "Léolo ". Le Parisien (16 septembre 1992).

Littamé, Fabrice. "John Malkovich un géant sur la Croisette». L’Union (18 mai 1992).

Loreau, Jean-François. "La bonne surprise du festival de Cannes. Léolo». La Gazette du cinéma (septembre 1992).

M., J.-L. «Le dompteur de rêves ». La Croix (18 septembre 1992).

Mallien, Jérôme. "Léolo: parce que moi je rêve...". Dernières Nouvelles d'Alsace (18 septembre 1992).

Mallien, Jérôme. "Le $45^{\circ}$ Festival de Cannes. Le palmarès sera connu ce soir. Léolo, l'émotion ". Dernières Nouvelles d'Alsace, n' 20 (18 mai 1992).

Maupin, Françoise. "Léolo, l'enfance nue». Figaroscope (16 septembre 1992).

Médioni, Gilles. «Léolo, l'enfant du frigo ". L'Express (12 septembre 1992) p. 148.

Nortier, Viviane. "Léolo. La passion selon Lauzon». La Dépêche du Midi, 16 septembre 1992.

P., F. "Les films qu'on peut voir. Léolo». Le Canard enchaîné (16 septembre 1992).

P., G. "Léolo de Jean-Claude Lauzon ”. La Vie (17 septembre 1992).

Parent, Denis. "L'écume des jours". Studio, n 63 (juin 1992).

Parra, Danièle. "Léolo de Jean-Claude Lauzon avec Maxime Collin, Julien Guiomar ". Le Cinéphage (septembre-octobre 1992). 
«Petit jeu des pronostics au $45^{\circ}$ Festival de Cannes». Presse-Océan et L'Éclair (18 mai 1992).

Philipponnat, Véronique. "Tous dingos chez Léolo ". Le Journal du dimanche (13 septembre 1992).

Rebichon, Michel, "Un film cru. Une enfance nue. Léolo de Jean-Claude Lauzon. Avec Maxime Collin, Ginette Reno... ». Studio, n 65 (septembre 1992) p. 22.

Rémy, Vincent. «Léolo». Télérama, n²227 (16 septembre 1992) p. 38.

Roig, Catherine et Françoise Delbecq. "Léolo". Une semaine avec Elle (14-20 septembre 1992).

Royer, Philippe. "Léo, l'affreux ". La Croix (19 mai 1992).

S., A. "Léolo". L'Officiel des spectacles (16 septembre 1992).

Seguret, Olivier. "Sélection officielle. Léolo ne l'est pas». Libération (18 mai 1992).

Sotinel, Thomas. "L'impolitesse du désespoir. Plongée dans le noir enfer des souffrances enfantines". Le Monde (18 septembre 1992).

"Spécial Canada. Les productions du Verseau. Léolo en Compétition officielle». Le Film français (17 mai 1992).

Toscan du Plantier, D. "Un air frais venu du Québec». Le Figaro magazine (19 septembre 1992).

Tran, David. «Beau comme Léolo». Le Progrès (16 septembre 1992).

Tranchant, Marie-Noelle. "Léolo. Jean-Claude Lauzon: l'enfance de l'art ". Le Figaro (17 septembre 1992).

Valot, Jacques. "Léolo". La Revue du cinéma (juin 1992).

Valot, Jacques. "Léolo roman familial ». La Revue du cinéma (septembre 1992).

Valot, Jacques. "Séquences cannoises". La Revue du cinéma (juin 1992).

Wais, Alain. "Léolo le Héros». Glamour (octobre 1992).

Whimphen, Catherine. "Léolo de Jean-Claude Lauzon». Studio (mai 1992). 